\title{
Prediction on Turbulent Flow of Two Parallel Plane Jets Using $\kappa-\varepsilon$ Model
}

\author{
Ramesh Chandra Mohapatra \\ Reader\& HOD, Mechanical Department, Government College of Engineering, Keonjhar, India \\ Email: rameshmohapatra75@gmail.com
}

How to cite this paper: Mohapatra, R.C. (2017) Prediction on Turbulent Flow of Two Parallel Plane Jets Using $\kappa-\varepsilon$ Model. Open Access Library Journal, 4: e3291. https://doi.org/10.4236/oalib.1103291

Received: December 10, 2016

Accepted: February 6, 2017

Published: February 9, 2017

Copyright (c) 2017 by author and Open Access Library Inc.

This work is licensed under the Creative Commons Attribution International License (CC BY 4.0).

http://creativecommons.org/licenses/by/4.0/

\begin{abstract}
In the present study, computational details of two parallel plane jets with nozzle separation ratio $=4.25$ and $\operatorname{Re}=11,000$ are presented. A study of the turbulence has also been done. The computational domain size, grid resolution, and different discretization schemes on the predictions are discussed. The existence of a recirculation flow region, a merging region and a combined region in the two parallel plane jets configuration has been predicted qualitatively by $\kappa-\varepsilon$ model. Power law scheme is used for discretizing the convective terms. Calculations were made using a Power law scheme. A code is used to solve a laminar, two dimensional viscous fluid flow and heat transfer. At the last it is shown that this code is ready to use for modification for mean flow solution of the turbulent two parallel plane jets. The numerical results are compared with the experimental results. Computational details of various mean flow and turbulent parameters are presented and described with separation ratio of 4.25 and $\operatorname{Re}=11,000$. The results are compared with Nasr and Lai and are found to be in good agreement with it. The two parallel plane jets develop like a single free jet in the combined region. The outer shear layer spreads faster than the inner shear layer in the near field.
\end{abstract}

\section{Subject Areas}

Mechanical Engineering

\section{Keywords}

Turbulent Flow, Separation Ratio, Power Law Scheme, $\kappa-\varepsilon$ Model, Reynolds Number, Plane Jets

\section{Introduction}

A jet is defined as a free or bounded one depending upon the distance of the confining boundaries. When the boundaries (parallel to inlet axis) are suffi- 
ciently away from the origin of the jet, it is termed as free jet. A bounded jet will occur when the flow interacts with a parallel wall. It can be classified into three types based on the orientation: (a) Impinging jet aimed toward the boundary; (b) Wall jet where fuid is discharged at the boundary; and (c) Offset jet from a vertical wall of a stagnent pool issuing parallel to a horizontal solid wall. Two parallel plane jets have numerous technological applications such as the gas turbine combustion chamber, the air conditioner unit for automobile, the air curtain unit for refrigerator system, entrainment and mixing processes in boiler, injection systems and so on. In environmental fluid mechanics, an optimum spacing between exhaust stacks (chimneys) is required to dilute disposal plums to a specified level within a given from the chimney. The details of the flow was studied by several authors. The first detail experimental study of the mean flow was reported by Tanaka [1] [2]. He described the basic flow patterns and entrainment mechanism of parallel jets. Elbanna et al. [3] showed that the mean velocity profile of the parallel jets agreed well with the single jet in the region downstream of the combined point. Lin et al. [4] [5] used hot-wire anemometry to show that the mean velocity approaches self-preservation in both the merging and combined regions, while Reynolds shear stresses approach self-preservation in the combined region only. Nasr et al. [6] [7] provided an experimental comparison between parallel, plane jets and an off set jet (where a wall replaces the symmetry plane). In a later work, they performed an experimental investigation into the effect of jet spacing on the mean stream-wise momentum flux measured at the combined point. Anderson et al. [8] presented experimental and numerical results for isothermal, plane parallel jets at spacings $/ \mathrm{w}=9,13$, and 18.25 (where s is the spacing between jet centre lines and $w$ is the jet width.). Computations of the two parallel plane jets performed by Militzer, J. [9] showed that the basic $\kappa-\varepsilon$ model does not take into account the effects of stream line curvature and hence led to quite an unreasonable prediction of flow characteristics. Lechziner et al. [10] considered both the effect of streamline curvature and preferential influence of normal stresses on the dissipation of turbulence energy. Nasr et al. [11] compared the obtained numerical results with experiment for the case of a turbulent plane offset jet.

In the present study, the mean velocity and static pressure field is presented for turbulent two parallel plane jets from the numerical study. The performance of the standard $k-\varepsilon$ turbulence model is evaluated for all three zones (converging zone, merging zone and combined zone) Computational details of two parallel plane jets with nozzle separation ratio $=4.25$ and $\mathrm{Re}=11,000$ are presented. Power law scheme is used for discretizing the convective terms. The results are compared with experimental results of Nasr and Lai and are found to be in good agreement with it.

\section{2. $\kappa-\varepsilon$ Turbulence Model}

$\kappa-\varepsilon$ Turbulence model is the most common model used in computational fluid dynamics (CFD) to simulate mean flow characteristics for turbulent flow 
conditions. It is a two equation model which gives a general description of turbulence by means of two transport equations to present the turbulent properties of the flow. This model solves for two variables: $\kappa$ - the turbulent kinetic energy and $\varepsilon$-the rate of dissipation of kinetic energy. The wall functions are used in this model, so the flow in the buffer region is not simulated. This model is very popular in industrial application due to its good convergence rate and relatively low memory requirements.

\section{Problem Specification}

The flow pattern of the two parallel plane jets is shown in Figure 1. Here, the two identical plane nozzles of width $\mathrm{w}$ are separated in the lateral $(y)$ direction by $s$, giving rise to a nozzle spacing ratio of $s / w$. The Cartesian coordinate system $(x y)$ is set so that its origin is at the nozzle plate and the $x$ axis is in the plane of symmetry which bisects the two nozzles. In general, the flow field can be classified into three parts: the converging region, where the reverse flow is created near the nozzle exit. In this region there is recirculation region where the mean stream wise velocity on the $x$ axis $\left(U_{c}\right)$ is negative. The merging region, where the velocity profile shows two local peaks without reverse flow. The two inner shear layers merge at the merging point $(\mathrm{mp})$ where $\left(U_{c}\right)$ is zero. Downstream from the merging point, in the merging region, the two jets continue to interact with each other and $\left(U_{c}\right)$ increases up to the combined point (cp) where $\mathrm{U}_{c}$ reaches a maximum value $\left(U_{\text {cmax }}\right)$. The combined region, where the velocity profile becomes similar to that of the single free jet. The stream-wise distances from the nozzle plate to the merging and combined points are referred to as merging length $\left(x_{\mathrm{mp}}\right)$ and combined length $\left(x_{\mathrm{cp}}\right)$ respectively. The essential feature of the flow is the entrainment of the surrounding fluid by turbulent jets, which causes a

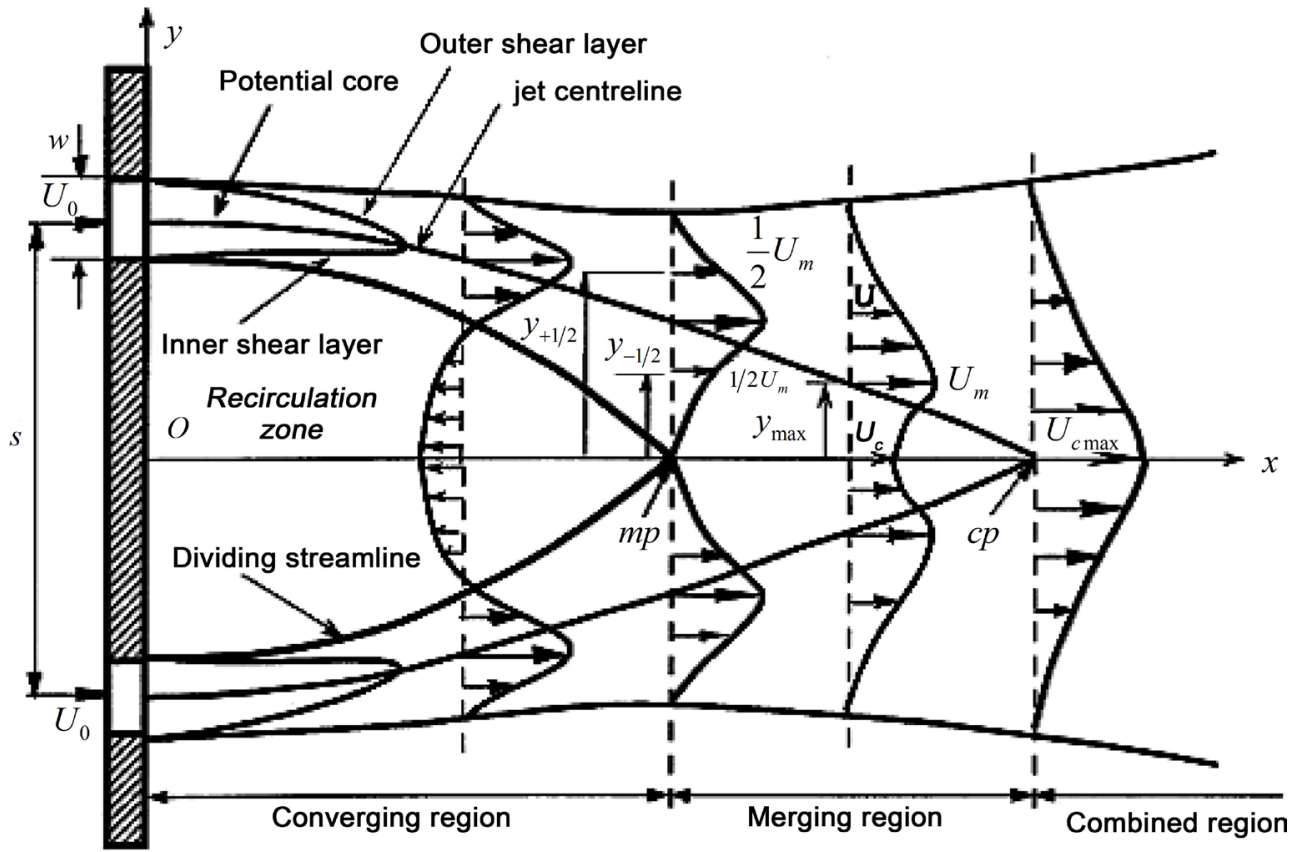

Figure 1. Diagram of two parallel plane jets. 
sub atmospheric pressure region between the jets near the nozzles. The jets draw each other and their trajectories deviate from straight lines because of mutual entrainment of the surrounding fluid.

\section{Governing Equations}

Continuity equation

$$
\frac{\partial \bar{U}}{\partial x}+\frac{\partial \bar{V}}{\partial y}=0
$$

$X$-Momentum equation

$$
\frac{\partial(\bar{U})^{2}}{\partial x}+\frac{\partial(\overline{U V})}{\partial y}=-\frac{1}{\rho} \frac{\partial \bar{P}}{\partial x}+\frac{\partial}{\partial x}\left[\left(v+v_{t}\right) \frac{\partial \bar{U}}{\partial x}-\frac{2}{3} \kappa\right]+\frac{\partial}{\partial y}\left[\left(v+v_{t}\right) \frac{\partial \bar{U}}{\partial y}\right]
$$

$Y$-Momentum equation

$$
\frac{\partial(\overline{U V})}{\partial x}+\frac{\partial(\bar{V})^{2}}{\partial y}=-\frac{1}{\rho} \frac{\partial \bar{P}}{\partial y}+\frac{\partial}{\partial x}\left[\left(v+v_{t}\right) \frac{\partial \bar{V}}{\partial x}\right]+\frac{\partial}{\partial y}\left[\left(v+v_{t}\right) \frac{\partial \bar{V}}{\partial y}-\frac{2}{3} \kappa\right]
$$

Assumptions made are:

1) Steady turbulence.

2) 2-D flow, flow variations in $z$-direction is neglected

3) Incompressible flow, i.e. dilation term is neglected.

4) Body forces are neglected.

5) Properties $(\mu, v, \rho)$ are assumed to be constant.

6) RANS equations are used for predicting the turbulent flow.

7) Boussinesq assumption is used to link Reynolds stress to velocity gradient.

8) $\kappa-\varepsilon$ Model is used for determining the turbulent viscosity.

\section{Non-Dimensional Equations}

Dimensionless variables are as follows

Non-dimensional velocity in $X$-direction $\overline{U_{n}}=\frac{\bar{U}}{U_{0}}$

Non-dimensional velocity in $Y$-direction $\overline{V_{n}}=\frac{\bar{V}}{V_{0}}$

Non-dimensional coordinates $X=\frac{\bar{x}}{h}$ and $Y=\frac{\bar{y}}{h}$

Non-dimensional pressure $P_{n}=\frac{p-p_{o}}{\rho U_{0}^{2}}$

Non-dimensional turbulent kinetic energy $\kappa_{n}=\frac{\kappa}{U_{0}^{2}} \kappa_{n}=\frac{\kappa}{U_{0}^{2}}$

Non-dimensional kinetic turbulent viscosity $v_{t, n}=\frac{v_{t}}{v}$

Continuity equation

$$
\frac{\partial \overline{U_{n}}}{\partial X}+\frac{\partial \overline{V_{n}}}{\partial Y}=0
$$

$X$-Momentum equation 


$$
\begin{aligned}
& \frac{\partial\left(\overline{U_{n}}\right)^{2}}{\partial X}+\frac{\partial\left(\overline{U_{n} V_{n}}\right)}{\partial Y} \\
& =-\frac{\partial\left(\frac{2}{3} \kappa+\bar{P}\right)}{\partial X}+\frac{1}{R_{e}} \frac{\partial\left[\left(1+v_{t, n}\right) \frac{\partial \overline{U_{n}}}{\partial X}\right]}{\partial X}+\cdot \frac{1}{R_{e}} \frac{\partial\left[\left(1+v_{t, n}\right) \frac{\partial \overline{U_{n}}}{\partial Y}\right]}{\partial Y}
\end{aligned}
$$

$Y$-Momentum equation

$$
\begin{aligned}
& \frac{\partial\left(\overline{U_{n} V_{n}}\right)}{\partial X}+\frac{\partial\left(\overline{V_{n}}\right)^{2}}{\partial Y} \\
& =-\frac{\partial\left(\frac{2}{3} \kappa+\bar{P}\right)}{\partial Y}+\frac{1}{R_{e}} \frac{\partial\left[\left(1+v_{t, n}\right) \frac{\partial \overline{V_{n}}}{\partial X}\right]}{\partial X}+. \frac{1}{R_{e}} \frac{\partial\left[\left(1+v_{t, n}\right) \frac{\partial \overline{V_{n}}}{\partial Y}\right]}{\partial Y}
\end{aligned}
$$

\section{Boundary Conditions}

Non dimensional boundary conditions are provided as input for solution. A uniform inlet velocity profile was set at the opening. The top and outlet boundaries were set at a constant pressure. The symmetry boundary condition was prescribed at the symmetry plane between the two jets and only half of the flow was simulated. For turbulent kinetic energy equation, boundary condition at nozzleexit is $\kappa_{n}=\frac{\kappa}{U_{0}^{2}}=1.5 I^{2}$ where $I$ a turbulent intensity is, and is equal to 0.02 .

\section{Results \& Discussions}

Code is run for two parallel plane jets with separation ratio of $s / w=4.25$. The domain length in the $x$ direction was varied from 15 to 30 nozzle widths whereas it was set at $10 \mathrm{w}$ or $15 \mathrm{w}$ in they direction. The grid size of $151 \times 101$ is considered for all cases. The standard $\kappa-\varepsilon$ turbulence model with a power law discretization scheme is used. Here the working fluid is air and Reynolds number is 11,000 . The results obtained from the code are compared with the experimental solution by Nasr and Lai.

\subsection{Mean Stream-Wise Velocity Distribution}

The distribution of the non-dimensional mean stream-wise velocity along the $\mathrm{x}$ axis $\left[\frac{U_{c}}{U_{0}}\right]$ is shown in Figure 2. It can be seen that the absolute magnitude of the negative velocity increases from the nozzle plate to a maximum and then decreases to zero at the merging point (mp).Downstream from the merging point, $\left[\frac{U_{c}}{U_{0}}\right]$ continues to increase up to the combined point where $\left[\frac{U_{c}}{U_{0}}\right]$ reaches maximum value. Downstream from the combined point, in the combined region, the two individual jets combine to resemble a single free jet flow. Mean stream-wise velocity along the plane of symmetry for $s / w=4.25$, is compared 


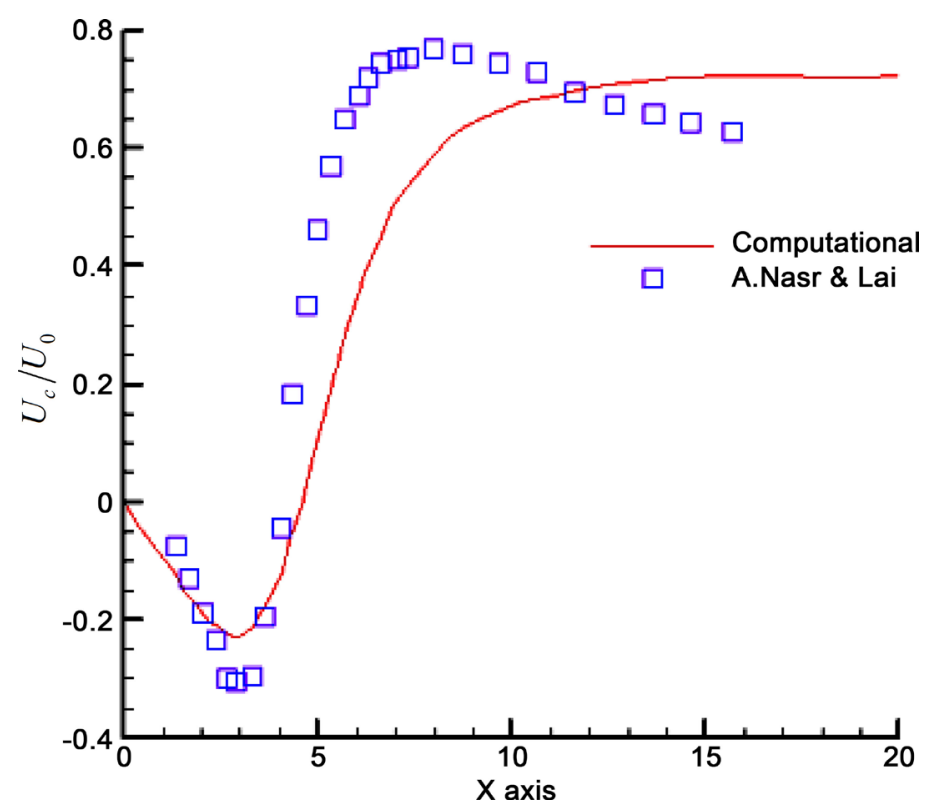

Figure 2. Mean stream-wise velocity distribution along the $x$ axis.

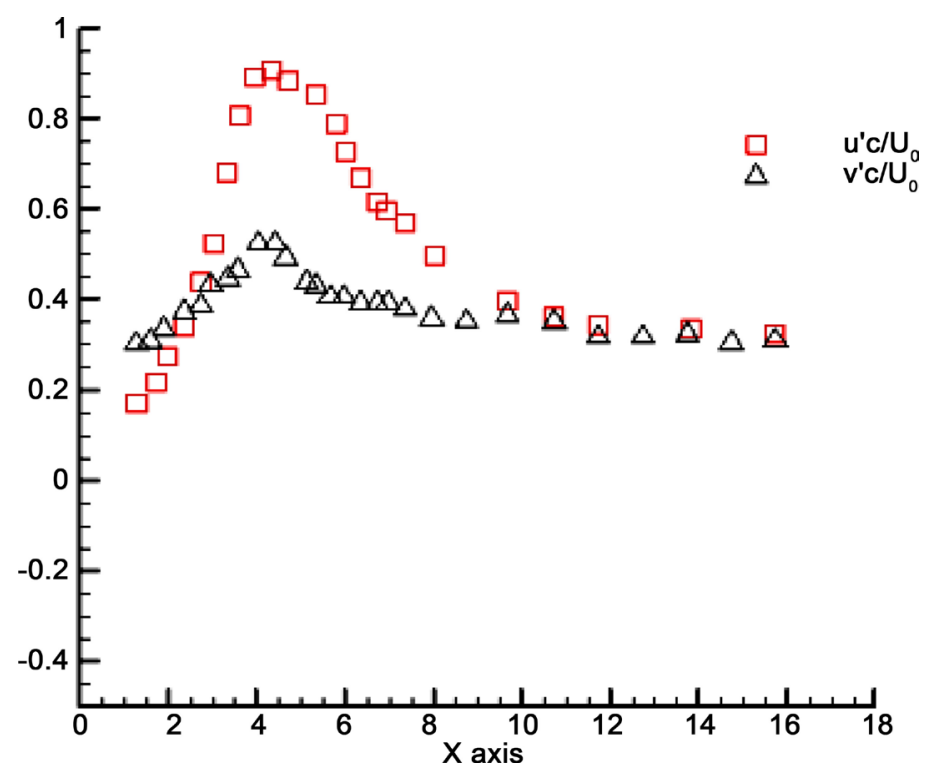

Figure 3. Turbulence intensities along the $x$ axis for the two parallel jets.

with the experimental results of Nasr and Lai. That shows the little difference in the deviation from the experimental results.

\subsection{Turbulence Intensities Distribution}

The distribution of lateral turbulence intensities are shown in Figure 3. This figure shows that $\frac{u_{c}^{\prime}}{U_{0}}$ and $\frac{v_{c}^{\prime}}{U_{0}}$ increase with $\mathrm{s} / \mathrm{w}$ to maximum near the merging point. It can also be observed that the maximum value of $\frac{u_{c}^{\prime}}{U_{0}}$ is relatively higher than $\frac{v_{c}^{\prime}}{U_{0}}$ because of the collision of the innershear layers from 
both sides of the plane symmetry and the large turning angle of the flow near themerging point.

\subsection{Maximum Velocity Point}

It is defined as the lateral distance from $x$ axis to the point where $U$ is equal to $U_{\max }$. Figure 4 shows that in the converging region $y_{\max }$ has been reasonably well predicted by $\kappa-\varepsilon$ model. However, in the merging and combined regions, there are substantial discrepancies between predictions by $\kappa-\varepsilon$ model.

\subsection{Locus of Half Velocity Points}

Half velocity points are the lateral distances from the $x$ axis to the points where the mean stream-wise velocity $(U)$ is half of its local maximum mean stream wise velocity $\left(U_{\max }\right)$ in the outer and innershear layers, respectively. Figure 5 shows that in the converging region, $y_{-\frac{1}{2}}$ and $y_{+\frac{1}{2}}$ have been reasonably well predicted by $\kappa-\varepsilon$ model as observed in Nasr results. However in the merging and combined region, $y_{+\frac{1}{2}}$ in the outer shear layer has been unpredicted. Furthermore, it is expected that as the inner shear layers of the two jets merge to form a single jet down stream of the merging point, $y_{\max }$ rapidly approaches $y_{-\frac{1}{2}}$.

\subsection{Static Pressure Distribution}

Figure 6 shows that for $\kappa-\varepsilon$ turbulence model, the static pressure decreases along the $x$ axis from the nozzle plane, reaches a minimum in the recirculation zone and then sharply increases to a maximum near the marging point, just as observed in Nasr results. It then decreases with $x$ and approches atmospheric pressure in the combined region for $\kappa-\varepsilon$ model.

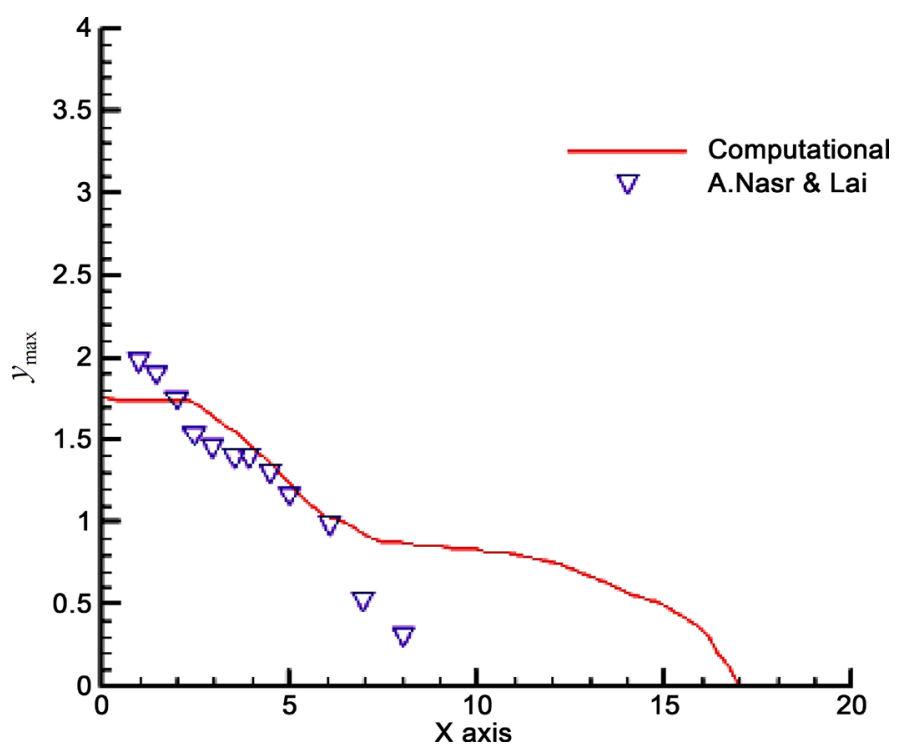

Figure 4. Comparison of maximum velocity points between computational and Nasr Lai results. 

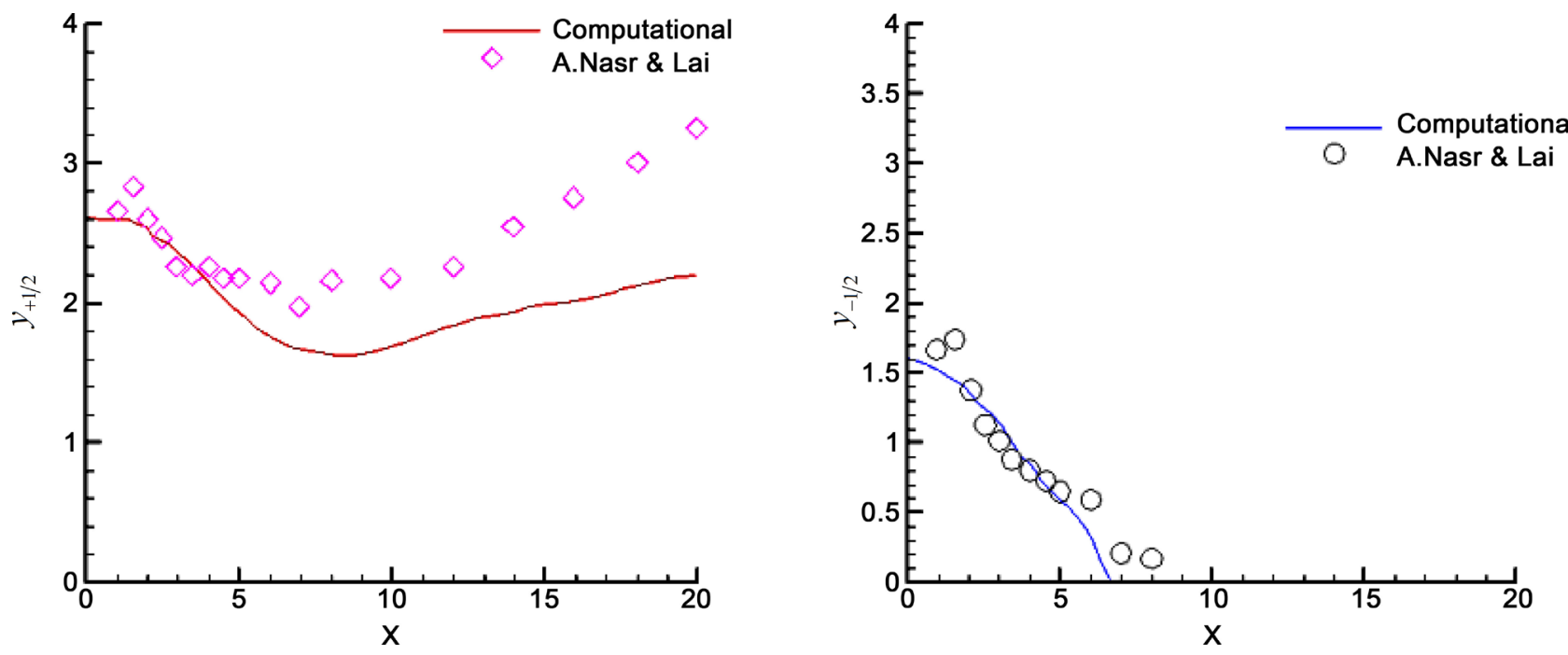

Figure 5. Comparison of half velocity points between computational and Nasr Lai results.

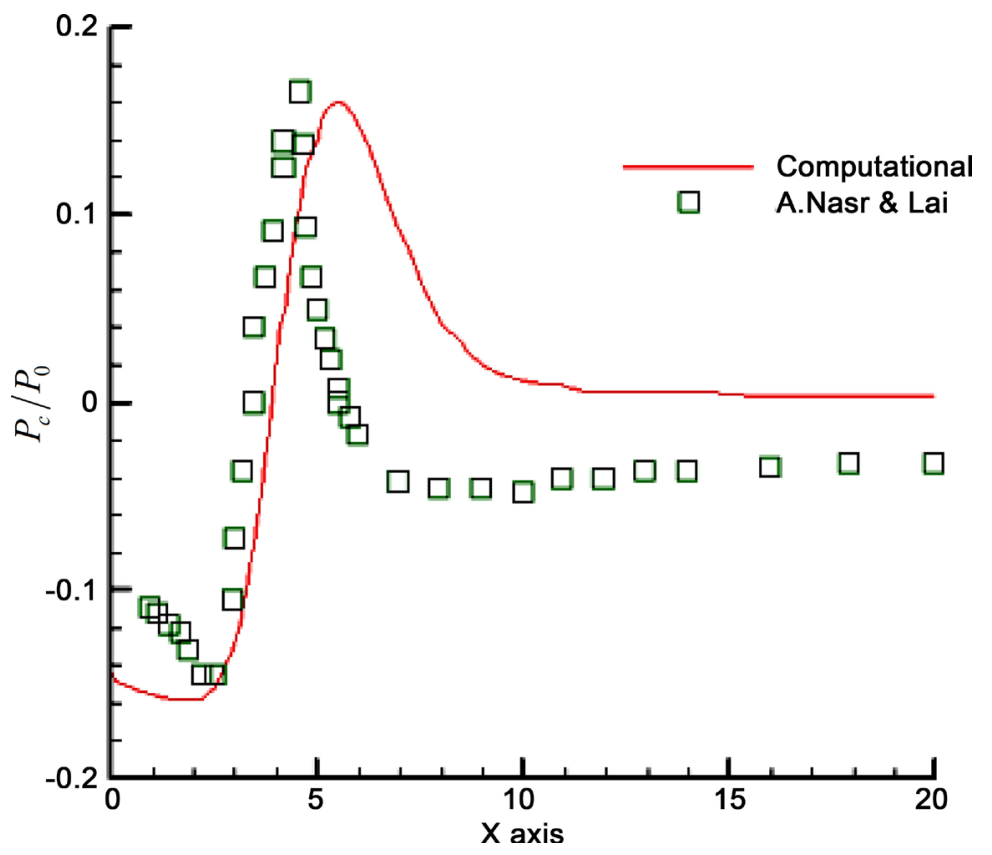

Figure 6. Comparison of static pressure between computational and Nasr and Lai.

\subsection{Static Pressure Contour}

The contour of static pressure computed by the $\kappa-\varepsilon$ model is shown in Figure 7. There is a sub-atmospheric zone in the region bounded by the nozzle plate, the inner shear layer and the symmetry plane. Downstream of the merging point, the static pressure increases to above atmospheric before recovering to atmospheric pressure further downstream in the combined region.

\subsection{Contour of Turbulence Kinetic Energy}

Contour of turbulence kinetic energy $\kappa$ computed by the $\kappa-\varepsilon$ model is shown in Figure 8. It indicates a much longer potential core and much slower spatial development for the jet. 


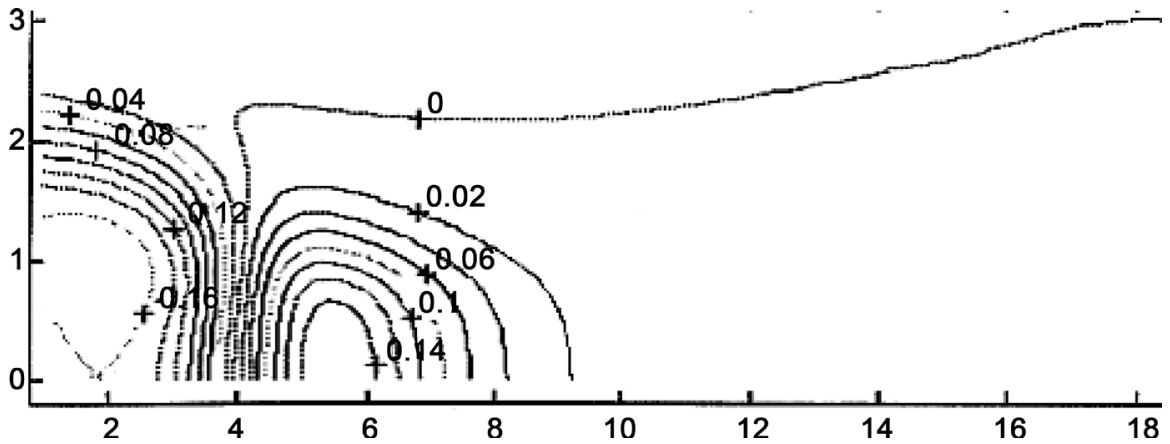

Figure 7. Static pressure contour.

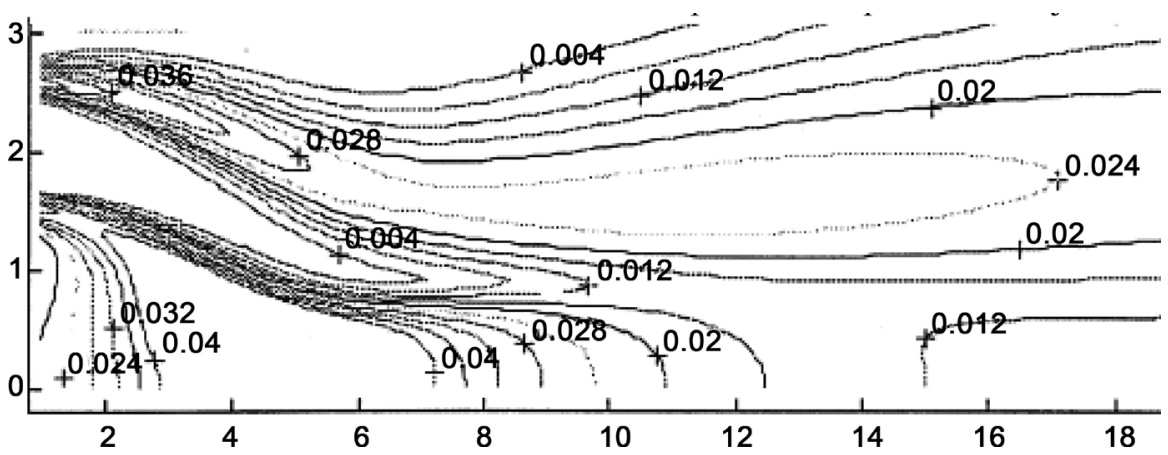

Figure 8. Turbulence kinetic energy for $\kappa-\varepsilon$ model.

\section{Conclusion}

The standard $\kappa-\varepsilon$ turbulence model for two parallel plane jets of a small nozzle separation ratio of 4.25 with a nozzle exit Reynolds number of 11,000 have been made. The recirculation flow, converging, merging and combined regions have been identified. The existence of negative and positive gauge static pressure zones on both sides of the merging point is also identified.

\section{References}

[1] Tanaka, E. (1970) The Interference of Two-Dimensional Parallel Jets (1st Report, Experiments on Dual Jet). Bulletin of JSME, 13, 272-280. https://doi.org/10.1299/jsme1958.13.272

[2] Tanaka, E. (1974) 2nd Report, Experiments on the Combined Flow of Dual Jet. Bulletin of JSME, 17, 920-927. https://doi.org/10.1299/jsme1958.17.920

[3] Elbanna, H., Gahin, S. and Rashed, M.I.I. (1983) Investigation of Two Plane Parallel Jets. AIAA Journal, 21, 986-990. https://doi.org/10.2514/3.8187

[4] Lin, Y.F. and Sheu, M.J. (1990) Investigation of Two Plane Parallel Unventileted Jets. Experiments in Fluids, 10, 17-22. https://doi.org/10.1007/BF00187867

[5] Lin, Y.F. and Sheu, M.J. (1991) Interaction of Parallel Turbulent Plane Jets. AIAA Journal, 29, 1372-1373. https://doi.org/10.2514/3.10749

[6] Nasr, A. and Lai, J.C.S. (1998) Comparison of Flow Characteristics in the Near Field of Two Parallel Plane Jets and an Offset Plane Jet. Physics of Fluids, 9, 2919-2931. https://doi.org/10.1063/1.869404

[7] Nasr, A. and Lai, J.C.S. (1999) Effects of Nozzle Spacing on the Development of Two Parallel Plane Jets. International Journal of Transport Phenomena, 2, 43-56. 
[8] Anderson, E.A. and Spall, R.E. (2001) Experimental and Numerical Investigation of Two Dimensional Parallel Jets. Journal of Fluids Engineering, 123, 401-406. https://doi.org/10.1115/1.1363701

[9] Militzer, J. (1977) Dual Plane Parallel Turbulent Jets. Ph.D. Thesis, University of Waterloo, Canada.

[10] Lechziner, M.A. and Rodi, W. (1981) Calculations of Annular and Twin Parallel Jets Using Various Discretization Schemes and Turbulence-Model Variations. ASME, Journal of Fluids Engineering, 103, 352-360. https://doi.org/10.1115/1.3241745

[11] Nasr, A. and Lai, J.C.S. (1998) A Turbulent Plane Offset Jet with Small Offset Ratio. Experiments in Fluids, 24, 47-57. https://doi.org/10.1007/s003480050149

Submit or recommend next manuscript to OALib Journal and we will provide best service for you:

- Publication frequency: Monthly

- 9 subject areas of science, technology and medicine

- Fair and rigorous peer-review system

- Fast publication process

- Article promotion in various social networking sites (LinkedIn, Facebook, Twitter, etc.)

- Maximum dissemination of your research work

Submit Your Paper Online: Click Here to Submit

Or Contact service@oalib.com 